 \\ LEUPHANA \\ UNIVERSITÄT LÜNEBURG
}

\section{Hybride}

Busch, Kathrin

Published in:

Kultureller Umbau

DOI:

10.14361/9783839405567-001

Publication date:

2007

Document Version

Verlags-PDF (auch: Version of Record)

Link to publication

Citation for pulished version (APA):

Busch, K. (2007). Hybride: der Raum als Aktant. in M. Kröncke (Hrsg.), Kultureller Umbau: Räume, Identitäten und Re/Präsentationen (S. 13-27). transcript Verlag. https://doi.org/10.14361/9783839405567-001

\section{General rights}

Copyright and moral rights for the publications made accessible in the public portal are retained by the authors and/or other copyright owners and it is a condition of accessing publications that users recognise and abide by the legal requirements associated with these rights.

- Users may download and print one copy of any publication from the public portal for the purpose of private study or research.

- You may not further distribute the material or use it for any profit-making activity or commercial gain

- You may freely distribute the URL identifying the publication in the public portal ?

Take down policy

If you believe that this document breaches copyright please contact us providing details, and we will remove access to the work immediately and investigate your claim. 


\title{
Hybride. Der Raum als AKtant
}

\author{
KATHRIN BUSCH
}

»Der Raum, aber Ihr könnt nicht begreifen, dieses fürchterliche Drinnen-und-Draußen, das der wahre Raum ist.« (Henri Michaux)

\section{Vom Akteur zum Aktant}

Die Philosophien des 20. Jahrhunderts, beginnend bei der Phänomenologie bis hin zu zeitgenössischen Sprach-, Symbol- und Medientheorien, verbindet bei allen Unterschieden das gemeinsame Anliegen, die neuzeitliche Gegenüberstellung von Subjekt und Objekt zu überwinden und ihr Getrenntsein als ein bloßes Konstrukt zu erweisen. In diesem Sinne argumentiert auch der Wissenschaftssoziologe Bruno Latour dafür, dass es ein reines, von den es umgebenden Dingen unberührtes Subjekt nie gegeben habe und vielmehr eine Vermischung der objektiven Realität mit subjektiven Vorstellungen oder der Dinge mit ihren Repräsentationen die Regel sei. ${ }^{1}$ Interessanterweise beschränkt Latour die Aufhebung der Trennung zwischen Subjekt und Objekt jedoch nicht auf Fragen der Wahrnehmung, Erkenntnis und Wirklichkeitskonstitution, sondern weitet seine These der Hybridisierung auf den Bereich der Praxis aus. Er fokussiert die Vermischung von Menschen und Dingen im Sinne einer Übertragung von Handlungspotentialen zwischen Subjekt- und Objektsphäre. Dabei besagt Hybridisierung nicht, auf die Objektwelt werde das Soziale projiziert - denn: »Objekte sind kein formloser Gegenstand für soziale Kategorien $~^{2}{ }^{2}$ - noch seien die Subjekte den objektiven Kräften schlichtweg unterworfen, vielmehr ist gemeint, dass sich Menschen und Dinge

1 Vgl. Bruno Latour: Das Parlament der Dinge. Für eine politische Ökologie, Frankfurt a.M.: Suhrkamp 2001, S. 237ff. Eine bündige Einführung in das Denken von Latour geben Nina Degele/Timothy Simms: »Bruno Latour (*1947). Post-Konstruktivismus pur«, in: Martin L. Hofmann/Tobias F. Korta/Sibylle Niekisch (Hg.): Culture Club. Klassiker der Kulturtheorie, Frankfurt a.M.: Suhrkamp 2004, S. 259-275.

2 Bruno Latour: Wir sind nie modern gewesen. Versuch einer symmetrischen Anthropologie, Frankfurt a.M.: Fischer 1998, S. 76. 
zu hybriden Netzwerken verflechten. ${ }^{3}$ Anders als bei Donna Haraway ist dabei weniger die technische Zurüstung und prothetische Verlängerung des menschlichen Körpers gemeint, ${ }^{4}$ als vielmehr Handlungsverbünde, an denen natürliche, technische und soziale Entitäten teilhaben und ineinanderspielen.

Mit dieser These widerspricht Latour zwei gegenläufigen Meinungen: zum einen der Vorstellung, dass die Menschen ihre Instrumente vollständig beherrschen und unberührt von ihnen über die Dinge nach eigenem Willen verfügen könnten und zum zweiten der ebenso verkürzenden Befürchtung, dass die Subjekte von einer eigengesetzlichen Dingwelt dominiert würden. Während die erste Position einem naiven Technikverständnis im Sinne eines bloßen Werkzeuggebrauchs entspringt, bleibt letztere nach Latour einem reduktionistischen Materialismus verhaftet. Die demgegenüber formulierte These der Hybridisierung stützt sich auf die Verschmelzung von Subjekt und Objekt qua Praxis. Zwischen beiden vermittelt ein Handlungsprogramm, das gleichermaßen die Eigendynamik der Objekte wie die Intentionen des Subjekts verändert und ablenkt, um sie zu einem neuen >Hybrid-Akteur $\mathrm{zu}$ synthetisieren, in dem sowohl Subjekt wie Objekt transformiert und ») jemand >etwas anderes werden. $\ll^{5}$

Anstatt also lediglich Subjekten die Rolle von Handelnden zuzubilligen, zeigt Latour, dass Handlungsfähigkeit wie Zielsetzungen sowohl menschlichen als auch nicht menschlichen Agenten zuzuschreiben sind. Daher habe man der Tatsache Rechnung zu tragen, »dass Gegenstände und andere nicht menschliche Wesen vollwertige Akteure in unserem Kollektiv sind $\varkappa^{6}$. Weil Techniken und Artefakte handlungsbestimmende Funktionen übernehmen und selbst als Handlungsträger fungieren, werden sie - in Anlehnung an die strukturalistische Terminologie - als Aktanten bezeichnet und weil sie in Handlungsverläufen eine mindestens ebenso große Rolle spielen wie die scheinbar allein agierenden Subjekte, lässt sich von einer »Symmetrie von Akteur und Aktant «" sprechen. Latour berücksichtigt außerdem, dass eine Handlung durch die Zusammen-

3 Vgl. Bruno Latour: »On actor-network theory. A few clarifications«, in: Soziale Welt 47 (1996), S. 369-381.

4 Vgl. Donna Haraway: »Ein Manifest für Cyborgs. Feminismus im Streit mit den Technowissenschaften«, in: dies.: Die Neuerfindung der Natur. Primaten, Cyborgs und Frauen, Frankfurt a.M./New York: Campus 1995, S. 33-72.

5 Bruno Latour: Die Hoffnung der Pandora. Untersuchungen zur Wirklichkeit der Wissenschaft, Frankfurt a.M.: Suhrkamp 2000, S. 218.

6 Ebd., S. 211.

7 Ebd., S. 219. 
setzung mehrerer Kräfte gebildet sein kann, in der neben dem Akteur verschiedene Aktanten - ganze Aktanten-Assoziationen - am Werk sind: »Handeln ist nicht das Vermögen von Menschen, sondern das Vermögen einer Verbindung von Aktanten. ${ }^{8}$ Diese Aktivität der Dinge und Techniken reicht nicht allein in das menschliche Handeln hinein, sondern vermag Bedeutungsfunktion zu übernehmen. Wenn Handlungsprogramme an Dinge delegiert werden, können diese für menschliche Akteure einstehen und Träger von sozialen Normen werden. ${ }^{9}$ Menschliche und nicht menschliche Wesen tauschen somit ihre Eigenschaften aus. Durch diese Hybridisierung von Subjekten und Objekten wird das soziale Gewebe auf nicht menschliche Wesen ausgedehnt, so dass man berechtigterweise mit Knorr Cetina von einer »Sozialität mit Objekten $«{ }^{10}$ sprechen kann. Wenn die Dinge am Sozialen partizipieren, so ist es nach Latour nur folgerichtig, dass heutzutage auch nicht menschlichen Wesen ein Rechtsstatus zugesprochen wird. ${ }^{11}$

Bedeutsam ist dieser Ansatz, weil er die These widerlegt, Gegenstände, Werkzeuge oder Techniken seien lediglich ein Resultat von Konstruktionen. Mit der verstärkten Berücksichtigung der Eigendynamik und Materialität von Dingen wendet er sich gegen konstruktivistische Theorieansätze, ohne jedoch in einen naiven Realismus zu verfallen.

\section{Interaktion mit Räumen}

Während Latour diese Hybridisierung vor allem in Bezug auf Dinge und Techniken im Blick hat, soll im Folgenden die Übertragung von Handlungspotentialen und -normen auf Raumordnungen und gebaute Räume einbezogen werden: Nicht lediglich die Gegenstände des Gebrauchs, sondern auch die gebauten Räume und räumlichen Anordnungen übernehmen handlungsstrukturierende Funktion. Hybridisierung betrifft dem-

8 Ebd., S. 221 (Hervorh. i. Orig.). Latour erläutert diese These am Beispiel des Flugverkehrs. Demnach wäre es verkürzend zu sagen, ein Mensch fliege, vielmehr sei Fliegen eine »Eigenschaft der gesamten Assoziation oder Verbindung von Entitäten, und dazu gehören Startpisten und Maschinen, Flughäfen und Ticketschalter«. (Ebd.)

9 Vgl. ebd., S. 226ff.

10 Vgl. Karin Knorr Cetina: „Sozialität mit Objekten. Soziale Beziehungen in post-traditionalen Wissensgesellschaften«, in: Werner Rammert (Hg.): Technik und Sozialtheorie, Frankfurt a.M./New York: Campus 1998, S. 83-120.

11 Vgl. Bruno Latour: Von der Realpolitik zur Dingpolitik oder Wie man Dinge öffentlich macht, Berlin: Merve 2005. 
nach nicht nur Mensch-Ding-Konstellationen, sondern lässt sich auf die Mensch-Raum-Relation ausdehnen. Latour selbst legt diese Übertragung nahe, ist doch eines der anschaulichen Beispiele, die er zur Stützung seiner These anführt, ein raumhaltiges Artefakt: Bodenschwellen, die der Verkehrsberuhigung dienen. ${ }^{12}$ Ihnen wird insofern Handlungspotential übertragen, wie sie das Verhalten von Autofahrern durch die Raumgestaltung und nicht durch Hinweisschilder steuern. Latour unterstreicht, dass dieser bauliche Eingriff Handlungsnormen transportiert und sie zugleich auf die dingliche Ebene verschiebt. Denn das Tempolimit wird nicht per Ordnung, sondern kraft Interaktion von Fahrer und Schwelle durchgesetzt. Es gibt nun eine ganze Reihe baulicher Vorgaben, die das alltägliche Verhalten steuern. Von der Wegeführung in Gebäuden bis hin $\mathrm{zu}$ architektonischen Anlagen, die ein Machtverhältnis einrichten und sicherstellen, wie Michel Foucault anschaulich anhand der Gefängnisarchitektur des 19. Jahrhunderts gezeigt hat. ${ }^{13}$ Die panoptische Architektur gibt ein gutes Beispiel für die Vermischung von Raum und Subjekt, hat Foucault doch aufgezeigt, wie mit dieser architektonischen >Maschineく ein spezifisches, auf Disziplinierung aufruhendes Selbstverhältnis allererst eingerichtet wird. Mit Recht kann man die panoptische Raumordnung als Aktanten der Disziplinarmacht bezeichnen, die sich auf andere Bereiche der Gesellschaft wie Schulen, Werkstätten, Fabriken oder Militär ausdehnend nicht nur das Verhalten steuert, sondern das moderne Subjekt in Form »nutzbringende[r] Individuen $«{ }^{14}$ fabriziert.

Ist mit diesem Beispiel angedeutet, wie sich Latours Konzept des Aktanten auf Fragen des Raumes berechtigterweise applizieren ließe, so ist mit ihr darüber hinaus das nötige argumentative Rüstzeug vorhanden, den konstruktivistischen Verkürzungen der derzeitig dominanten Raumtheorien entgegenzuarbeiten. Überträgt man Latours Hybridisierungsthese auf den Raum, dann lässt sich damit die seit einigen Jahren in die Kritik gekommene Vorstellung vom homogenen Behältnisraum in ihrer Fragwürdigkeit bestätigen. Zugleich wird aber auch die sich stattdessen etablierende Theorie der Produktion des Raumes in ihrer problematischen Vereinseitigung sichtbar.

In den Kulturwissenschaften ist mit dem sogenannten spatial turn ${ }^{15}$ die Abkehr von einem betrachterunabhängigen, statischen sowie bedeu-

12 Vgl. B. Latour: Die Hoffnung der Pandora, S. 226ff.

13 Vgl. Michel Foucault: Überwachen und Strafen. Die Geburt des Gefängnisses, Frankfurt a.M.: Suhrkamp 1989, S. $251 \mathrm{ff}$.

14 Ebd., S. 271.

15 Foucault war einer der Ersten, der die inzwischen unter dem Schlagwort spatial turn firmierende These vertreten hat, dass wir in einer Epoche des Raumes leben, in der nicht mehr die Zeit, sondern Verortung und Nebenei- 
tungsneutralen Raumbegriff vollzogen und zugleich die Aufmerksamkeit auf die verschiedenen kulturellen und sozialen Formierungen von Raum gelenkt worden. In Abgrenzung zur geometrischen, euklidischen Raumvorstellung wird in diesen Theorieansätzen Raum nicht länger als eine den Dingen vorhergehende Entität verstanden. Die Vorstellung vom Raum als homogenem, leerem sowie starrem Behältnis für Dinge oder Körper wird zugunsten unterschiedlicher Entwürfe eines dynamischen, sich wandelnden und relationalen Raums abgelöst. Nicht nur die moderne Physik reflektiert die Relativität des Raums, sondern auch in der Philosophie gewinnt der innere, der gelebte sowie der sozial geschaffene Raum zunehmend an theoretischer Beachtung. In den sich damit durchsetzenden relationalen oder topologischen Theorien wird Raum als etwas angesehen, das sich allererst aus der relativen Situierung von Körpern ergibt. In dem Maße aber, wie sich Raum über Lageverhältnisse und Ortsrelationen bestimmt, wird auch die These der Produktion von Raum durch Verortungen und Handlungen einsichtig. Damit rückt nun aber in der gleichen Weise die Eigengesetzlichkeit und Wirksamkeit von Räumen in den Hintergrund, wie in konstruktivistischen Theorieansätzen die Mitwirkung der Objektwelt vernachlässigt wurde.

So wird beispielsweise in der derzeitigen Diskussion um eine Neufassung des Raumbegriffs vielfach dafür argumentiert, dass die Konstitution des Raumes als ein sozialer Prozess zu gelten hat. ${ }^{16}$ Der Raum und die Raumordnung wird, etwa im Rückgriff auf Simmel, als Form und Medium der Vergesellschaftung gedacht, in dem sich das Soziale offenbart. ${ }^{17}$ Auch Bourdieu argumentiert für eine Parallelität des sozialen mit dem »angeeigneten, physischen Raum«, insofern der soziale Raum »die Tendenz auf[weist], sich mehr oder weniger strikt im physischen Raum in Form einer bestimmten distributionellen Anordnung von Akteuren und Eigenschaften niederzuschlagen $\ll{ }^{18} \mathrm{Da}$ sich der soziale im physischen

nander von bestimmender Bedeutung sei. Vgl. Michel Foucault: »Andere Räume«, in: Karlheinz Barck u.a. (Hg.): Aisthesis. Wahrnehmung heute oder Perspektiven einer anderen Ästhetik, Leipzig: Reclam 1990, S. 34-46.

16 Vgl. Martina Löw: Raumsoziologie, Frankfurt a.M.: Suhrkamp 2001, S. 67.

17 Vgl. Georg Simmel: »Der Raum und die räumlichen Ordnungen der Gesellschaft«, in: ders.: Soziologie. Gesamtausgabe Bd. 11, Frankfurt a.M.: Suhrkamp 1992, S. 687-790. Simmel differenziert die verschiedenen Raumqualitäten und arbeitet deren Bedeutung für die Form einer Gesellschaft heraus.

18 Pierre Bourdieu: »Physischer, sozialer und angeeigneter Raum«, in: Martin Wentz (Hg.): Stadt-Räume, Frankfurt a.M.: Campus Verlag 1991, S. 25-34, hier S. 26. Die Einschreibung der Strukturen der sozialen Ordnung voll- 
Raum realisiert oder in diesen eingelagert ist, ließe sich der bloß physische Raum nur anhand einer Abstraktion denken, nämlich unter Absehung dessen, dass er »eine soziale Konstruktion und eine Projektion des sozialen Raumes, eine soziale Struktur in objektiviertem Zustand ${ }^{19}$ sei. Wenn Bourdieu belegen kann, dass und auch wie sich das Soziale in den >physischen « Raum einschreibt, so berücksichtigt er jedoch nicht, inwiefern räumliche Anordnungen ihrerseits strukturierende Wirkungen ausüben. Dass der Raum nicht nur gesellschaftlich produziert ist, sondern auch eigene Wirksamkeiten im Umgang mit ihm entfaltet, gerät aus dem Blick.

Ein ähnlicher Befund ergibt sich im Hinblick auf die Raumkonzepte, die für die Produktion von Raum durch raumbezogene Handlungen argumentieren. ${ }^{20}$ Neben dem Raum als Produkt sozialer Beziehungen rücken hier die verschiedenen kulturellen und künstlerischen Praktiken der Raumbildung durch Aneignung und Umnutzung von Raum in den Blick. ${ }^{21}$ Was auch hierbei vernachlässigt wird, sind die Grenzen der Konstruier- und Beherrschbarkeit von Raum. Die bestimmenden Kräfte sowie die Eigendynamik der jeweiligen Raumanordnungen werden nur allzu oft ausgespart, wenn Räume lediglich als Signatur von Praktiken Bedeutung erlangen.

Wenn in den Raumtheorien des 20. Jahrhunderts gegen die Vorstellung argumentiert wird, der Raum existiere wie ein Behältnis unabhängig vom Handeln der in ihm befindlichen Subjekte, dann ist damit also noch keineswegs eine Symmetrie von Akteur und Aktant im Sinne von Latour erreicht. Vielmehr tendieren sie zu einer Vereinseitigung zugunsten des Subjekts, insofern seine Handlung mit der Produktion von Räumen einhergeht und die bestimmende Kraft von Räumen in Vergessenheit gerät. Diese problematische Überbetonung der Herstellung, Aneignung und Umnutzung von Raum lässt sich nun aber durch die These vom Raum als Aktant korrigieren, der zum einen nicht unberührt von sozialen Strukturen und menschlicher Aktivität zu denken ist und zum anderen seinerseits in die menschlichen Handlungen ein- und auf sie übergreift. Räume

zieht sich vermittels Körpertechniken, die über Raumstrukturen organisiert werden.

19 Ebd., S. 28.

20 Theoretische Bezugspunkte sind hier vor allem Henri Lefèbvre: Production de l'espace, Paris: Anthropos 1974 und Michel de Certeau: Kunst des Handelns, Berlin: Merve 1988.

21 Zur Raumproduktion der Kunst seit den 1960er Jahren vgl. Nina Möntmann: Kunst als sozialer Raum, Köln: Verlag der Buchhandlung Walther König 2002. 
sind sowohl durch Praktiken und gesellschaftliche Strukturen konstituiert wie sie ihrerseits Handlungsvollzüge mitbestimmen. ${ }^{22}$

Der Einsatzpunkt bei einer Theorie des Aktanten bietet damit den Vorteil, nicht nur die Performanz von Räumen, sondern auch die Symmetrie von Mensch und Raum zu begründen. Ebenso wenig wie natürliche Objekte oder Artefakte als bloße Projektionsflächen subjektiver Vorstellungen oder sozialer Konstruktionen fungieren, ist der Raum lediglich ein menschliches Konstrukt. Es ist daher möglich, mit dieser Übertragung des Aktanten-Modells auf Räume, deren nicht deterministische Wirksamkeit zu bedenken. Weder existiert der Raum unabhängig vom Subjekt noch produziert der Mensch asymmetrisch durch sein Handeln den Raum. Vielmehr ist auch hier eine Hybridisierung zu denken, in der Akteur und Aktant sich symmetrisch ergänzen. Dies heißt nun aber nach Latour, dass man nicht von zuvor konstituierten Entitäten auszugehen habe, die sich nachträglich vermischen, sondern dass bereits im Prozess der Konstituierung die Hybridisierung der jeweiligen Komplemente ansetzt.

\section{Hybridisierung von Subjekt und Raum}

Es gibt nun innerhalb des philosophischen Raumdenkens einen Strang von Theorien, die dieses Zusammenwirken von Mensch und Raum theoretisieren. Es sind Ansätze, die - dem phänomenologischen Ansinnen verpflichtet, das Begegnende nicht als gegenständliches Etwas, sondern im >Wier seines Gegebenseins auffassen - die Wahrnehmung des Rauumes fokussieren. Ausgehend von der konkreten leiblichen Erfahrung wird die homogene Räumlichkeit zugunsten des gelebten Raumes relativiert, der Qualitäten, Ausrichtungen und Orientierungen aufweist und mit Bedeutsamkeiten besetzt ist. Der Vorteil dieser Theorien besteht unzweifelhaft darin, den Begriff eines absoluten, vom Wahrnehmenden unabhängigen Raumes als fälschlich zu erweisen. Das Subjekt befinde sich nicht im Raum wie in einer Schachtel, sondern Raum und Subjekt bedingen sich gegenseitig und bilden sich korrelativ heraus. So hat etwa Merleau-Ponty mit dem Begriff der Tiefe angedeutet, dass das Subjekt selbst an der Szenerie teilhat, in der sich die Erfahrung des Raumes entfaltet. ${ }^{23}$

22 In diesem Sinne argumentiert auch die Soziologin Martina Löw: »Raum ist demnach gesellschaftlich produziert, entfaltet jedoch eine eigene Wirkung im Kontext menschlicher Nutzung.« (M. Löw: Raumsoziologie, S. 138)

23 Vgl. Maurice Merleau-Ponty: »Das Auge und der Geist«, in: ders.: Das Auge und der Geist. Philosophische Essays, Hamburg: Meiner 1984, S. 1343. 
Diese leibliche Teilhabe ist Grund einer anfänglichen Verflechtung von Raum und Subjekt. Dennoch muss man vor allem zwei Kritiken an den phänomenologisch geprägten Raumtheorien gelten lassen: Zum Ersten entgehen ihnen, weil sie von den Gegebenheiten der Wahrnehmung und des Erlebens ausgehen, die den Räumen innewohnenden Machtkonstellationen. Zum Zweiten verkennen sie, dass diese ihrerseits auf den Körper disziplinierend zugreifen und auch das leibliche Selbstverhältnis in einer spezifischen, historisch variablen Weise einrichten. Trotz dieser Einwände ist den phänomenologischen Ansätzen zugutezuhalten, eine Dimension der Raumerfahrung aufzudecken, in der Mensch und Raum ineinandergreifen und die Eigenmächtigkeit des Raumes sowie die Raumerfahrung so miteinander verzahnt sind, dass man berechtigterweise von einer Hybridisierung von Subjekt und Umraum sprechen kann. ${ }^{24}$

In aller Deutlichkeit zeigt sich diese Hybridisierung von Mensch und Umraum in der unter dem Begriff des >dunklen` oder >gestimmten Raumes firmierenden Raumtheorie. Sie setzt beim Raumempfinden an, wendet sich der atmosphärischen Übertragung von Raumcharakteren zu und gipfelt - bei Caillois und Lacan - in der These einer regelrechten »Verführung durch den Raum ${ }^{25}{ }^{25}$ Die Nachwirkungen dieser ursprünglich vor allem im Umkreis der Psychopathologie von Straus, Binswanger und Minkowski ${ }^{26}$ entwickelten Theorien eines affektiv auf das Subjekt übergreifenden Raumes lassen sich - vermittelt über Merleau-Ponty ${ }^{27}$ bis in die Texte von Foucault ${ }^{28}$ und de Certeau ${ }^{29}$ nachweisen. Sie widmen

24 Zur neueren phänomenologischen Raumtheorie vgl. Edward S. Casey: »Vom Raum zum Ort in kürzester Zeit. Phänomenologische Prolegomena«, in: Phänomenologische Forschungen (2003), S. 55-95.

25 Roger Caillois: »Mimetismus et psychasthénie légendaire«, in: ders.: Le Mythe et l'homme, Paris: Edition Gallimard 1938; Lacan übernimmt Caillois' These der Mimikry, um die unbewusste Dimension des Raumes zu ermessen. Vgl. Jacques Lacan: Das Seminar. Buch XI (1964), Berlin/Weinheim: Quadriga 1987, insb. S. 80 u. $104 \mathrm{ff}$.

26 Erwin Straus: »Die Formen des Räumlichen«, in: ders.: Psychologie der menschlichen Welt, Berlin u.a.: Springer Verlag 1960, S. 141-178; Ludwig Binswanger: »Das Raumproblem in der Psychopathologie«, in: Zeitschrift für Neurologie 145 (1933), S. 598-647; Eugène Minkowski: »Ansätze zu einer Psychopathologie des Raumes«, in: ders.: Die gelebte Zeit, Bd. 2, Salzburg: Otto Müller Verlag 1972, S. 232-267.

27 Bei Merleau-Ponty findet sich eine philosophische Aneignung und Aufarbeitung der aus der Psychopathologie stammenden Texte zum dunklen Raum. Vgl. Maurice Merleau-Ponty: Phänomenologie der Wahrnehmung, Berlin: de Gruyter 1966, S. 326ff.

28 Nach Foucault antwortet das sich im 18. Jahrhundert etablierende Paradigma der Überwachung und Disziplinierung auf eine Furcht vor dem 
sich der atmosphärischen Ausstrahlung von Räumen und der bisweilen beunruhigenden Tatsache, dass sich der erlebte Raum in die körperliche Zuständlichkeit überträgt, so dass die Grenze zwischen umgebendem Raum und Subjekt porös wird und damit die vereinnahmenden Kräfte des Raumes zutage treten.

Wenn man also im Sinne von Latour eine Wechselwirkung von Mensch und Raum geltend machen kann, dann, weil der umgebende Raum kein passives Objekt ist, sondern sich zwischen Subjekt und Umraum eine hybride Sphäre der Interferenz ausbildet. So wie sich Soziales und Kulturelles verräumlichen, so kommt den Räumen ihrerseits eine bestimmende Kraft in Bezug auf die in ihnen befindlichen Subjekte zu. Impliziert Latours These der symmetrischen Interaktion von Subjekt und Objekt, dass die Dinge keine bloß passiven Objekte sind, so bedeutet dies im Umkehrschluss allerdings auch, dass Menschen nicht ausschließlich aktive Subjekte sind. Oder anders gesagt: In gleichem Maße, wie die als passiv geltende Dingwelt mit Aktionspotential ausgestattet wird, ist dem passiven Bestimmtwerden der Subjekte Rechnung zu tragen. Dieses Bestimmtwerden betrifft nun aber, wie über die phänomenologischen Untersuchungen zum Raumerleben angedeutet werden sollte, nicht ausschließlich den Bereich der Praxis. Die raumhaltigen Artefakte verfügen neben Handlungspotentialen in nicht zu vernachlässigendem Maße über atmosphärische Qualitäten, die sich auf seine Bewohner übertragen. Dies resultiert aus dem Umstand, dass das Subjekt dem Raum nicht gegenübersteht, sondern in ihn eingelassen ist und durch ihn in seiner Befindlichkeit bestimmt wird.

Latour, der hinsichtlich seiner Überwindung des Subjekt-ObjektDualismus auf Heidegger rekurriert, hätte der Daseins-Analyse aus Sein und Zeit entnehmen können, dass dem Menschen durch seine Verstricktheit in die Welt diese nicht nur praktisch und bedeutungshaft, sondern

dunklen Raum. Als ») Gegenfigur zu den Transparenzen und zu den Sichtbarkeiten« findet sich lediglich in den Schauerromanen dieser Zeit das aus der neuen Ordnung des Raumes Ausgeschlossene artikuliert. In den Imaginationen finsterer Räume wird thematisiert, was zugunsten der »Macht durch Transparenz« verschwinden muss. Vgl. Michel Foucault: »Das Auge der Macht«, in: ders.: Schriften, Dritter Band, Frankfurt a.M.: Suhrkamp 2003, S. 250-271, hier S. 257ff.

29 Certeaus Abgrenzung gegenüber dem panoptischen Raum zielt auch auf eine Wiedergewinnung des erlebten, dunklen Raumes, wobei Certeau diese andere Räumlichkeit stärker aktivisch, nämlich ausgehend vom Umgang mit dem Raum statt pathisch, d.h. vom Raumempfinden aus entwickelt gleichwohl sind die Bezüge auf Merleau-Ponty unübersehbar. Vgl. M. de Certeau: Kunst des Handelns, S. 182 und 205f. 
auch stimmungshaft erschlossen ist. ${ }^{30}$ Diese Dimension, nicht bloß der Interaktion von Mensch und Raum, sondern ihrer - wie man formulieren könnte - Interpassion, wird von Latour hinsichtlich der gesamten Dingwelt ausgespart und verrät sein Verhaftetsein im Handlungsparadigma, das für die neuzeitliche Philosophie ebenso signifikant ist wie die Subjekt-Objekt-Dualität. Wenn mit der Hybridisierung von menschlichem Akteur und nicht menschlichen Aktanten berechtigterweise bedacht wird, dass in Handlungsvollzügen Kräfte im Spiel sind, durch die das Subjekt bis in seine Handlungsintentionen affiziert wird, so blendet Latour gleichwohl aus, dass die Verstrickung von Subjekt und Objekt in den Bereich der Empfindungen und Atmosphären hineinreicht. Die These der Hybridisierung von Subjekt- und Objektsphäre greift in der ausschließlichen Orientierung am Handlungsparadigma zu kurz und soll daher durch den Aspekt der Interpassion im Sinne der Affektion ergänzt werden.

\section{Von der Interaktion zur Interpassion}

In neueren Architekturtheorien findet die affektive Dimension des Raumes einige Beachtung. Im Rückgriff vor allem auf Gernot Böhmes Ästhetik der Atmosphären ${ }^{31}$ wird für eine verstärkte Berücksichtigung der affektiven Kraft von Räumen argumentiert. Folgerichtig verbindet sich diese Ausrichtung an Gefühl und Empfindung mit Theorien der Performativität, ${ }^{32}$ um die Aufmerksamkeit auf das zu lenken, was gebaute Räume bewirken, was sie einrichten und realisieren und weniger, was sie bedeuten oder repräsentieren. Zwar darf nicht vernachlässigt werden, dass räumliche Performanz auch beinhaltet, dass Architekturen als Herrschaftszeichen fungieren und zur Etablierung von Machtdispositiven dienen können, das theoretische Interesse fokussiert sich derzeit jedoch auf die Affekt-Übertragungen von Gebäuden. ${ }^{33}$ Die Atmosphäre von Räu-

30 Vgl. die Ausführungen zu Befindlichkeit und Stimmung in: Martin Heidegger: Sein und Zeit, Tübingen: Niemeyer 1986, S. $28 \mathrm{ff}$.

31 Vgl. Gernot Böhme: Atmosphäre. Essays zur neuen Ästhetik, Frankfurt a.M.: Suhrkamp 1995.

32 Vgl. beispielsweise Jens Roselt: »Wo die Gefühle wohnen. Zur Performativität von Räumen«, in: Hajo Kurzenberger/Annemarie Matzke (Hg.): Theorie, Theater, Praxis, Berlin: Theater der Zeit 2004, S. 66-76.

33 Zur Entwicklung des Zusammenhangs von Raum und Affekt in der Geschichte der Architekturtheorie vgl. Ullrich Schwartz: »Space Body Affect«, in: Tom Fecht/Dietmar Kamper (Hg.): Umzug ins Offene. Vier Versuche über den Raum, Wien/New York: Springer 2000, S. 82-85 und Niko- 
men erscheint nicht mehr als bloß dekorative Zutat, sondern wird zum eigentlichen Medium der Wirksamkeit von Raum. Im Hinblick auf gebaute Räume vermittelt sich die affektive Wirkung leiblich und schreibt sich über die Involvierung des Körpers bis in habitualisierte Lebensformen ein. Dabei übertragen sich Atmosphären subkutan, bleiben unterhalb der bewussten Wahrnehmung und lassen sich kaum dingfest machen. Dies heißt allerdings nicht, dass sie nicht geschichtlich kodiert oder historisch wandelbar wären. So richtig es ist, dass Atmosphären Überschussphänomene sind, will sagen: sich gegenüber einer sprachlichen Erfassung und Bedeutungsentschlüsselung sperren, so falsch wäre es, ihnen aufgrund dieses Umstandes Unmittelbarkeit zu attestieren. ${ }^{34}$ Die angesichts des Atmosphärischen gängige Rede von Unmittelbarkeit und Präsenz ist grob verfälschend, schließt sie doch sowohl die kulturelle wie soziale Konstitution der Raumwahrnehmung aus. Auch hinsichtlich der Wahrnehmung von Atmosphären bedarf es spezifischer Befähigungen der Rezeption, die über Kontexte und Diskurse vermittelt werden. Räumlich inszenierte Atmosphären unterliegen gleichermaßen ästhetischen Kodierungen, Moden oder Konventionen wie beispielsweise bildliche Repräsentationen und wirken keineswegs unmittelbar. Allerdings verstärkt die spezifisch räumliche Situation - im Unterschied zum gegenständlichen oder bildlichen Gegenüber - in ihrer Qualität als Umgebung den Eindruck einer nicht zu distanzierenden Ansteckung und affektiven Involvierung. Dieses Phänomen der Eintauchung in den umgebenden Raum, das als ausgezeichneter Fall der angedeuteten Interpassion gelten kann, wird derzeit unter dem Begriff der Immersion ${ }^{35}$ diskutiert.

laus Kuhnert/Anh-Linh Ngo: »Die Produktion von Präsenz. Potenziale des Atmosphärischen«, in: archplus 178 (2006), S. 22-25.

34 Gerade die Unbestimmtheit von Atmosphären kann als Indiz dafür gewertet werden, dass sich in ihre Wahrnehmung die Erfahrung von NichtPräsentischem, Abwesendem, Verborgenem oder bloß Angedeutetem oder auch Potentiellem mischt. Gerade die neuere Architektur hat sich unter dem Titel einer projektiven Architektur dieser Idee der Inszenierung des Möglichen verschrieben und setzt auf den Entwurfscharakter der Architektur - nicht als Vorstufe des Gebäudes, sondern als Potentialität fertiger Bauten. Gemeint ist die bauliche Umsetzung von Dispositionen, die zu unvorhergesehenen Verhaltensformen anregen und damit neue Lebensformen andeuten und nahelegen können. Vgl. Ole W. Fischer: »Critical, PostCritical, Projective«, in: archplus 174 (2005), S. 92-97.

35 Sloterdijk definiert Immersion als ein Verfahren der Entgrenzung von Bildern zu Umgebungen, in die man eintauchen kann. Vgl. Peter Sloterdijk: »Architektur als Immersionskunst«, in: archplus 178 (2006), S. 58-61, hier S. 58 . 
Implizit und unbeabsichtigt hat Fredric Jameson in seinem viel gelesenen Aufsatz Postmoderne - zur Logik der Kultur im Spätkapitalismus eine treffende - allerdings kritisch gemeinte - Beschreibung einer Raumerfahrung gegeben, die man heute mit dem Begriff des Immersion belegen würde. Ausgehend von der Bemerkung, dass unsere heutige Kultur zunehmend vom Raum und von räumlicher Logik dominiert wird, beschreibt er ganz in Übereinstimmung mit unseren Überlegungen zur Hybridisierung von Raum und Subjekt, dass wir in die Veränderungen der Architektur einbezogen sind und inwiefern sich unsere Wahrnehmungsfähigkeit und Handlungsformen angesichts dieser neuen Räume zu modifizieren haben. Jamesons Hauptthese besagt, dass es dem postmodernen Raum gelungen sei, »die Fähigkeit des individuellen menschlichen Körpers zu überschreiten, sich selbst zu lokalisieren, seine unmittelbare Umgebung durch die Wahrnehmung zu strukturieren und kognitiv seine Position in einer vermessbaren äußeren Welt durch Wahrnehmung und Erkenntnis zu bestimmen «. ${ }^{36}$ Jameson entwickelt diese These anhand einer eingängigen Beschreibung des 1977 von John Portman in Los Angeles erbauten Bonaventure-Hotels. Dieses Gebäude bilde ein unübersichtlich gewordenes Raumgefüge aus, in das man als Benutzer ohne distanzierende Wahrnehmungsmöglichkeit eingelassen sei. Anstatt den Raum überblicken und zumindest visuell einnehmen zu können, werde man von seiner Größe, Unübersichtlichkeit sowie seinen mobilen Elementen ergriffen. Das $»$ Eintauchen in den Raum $\aleph^{37}$ werde durch die verwirrende, nicht überschaubare, desorientierende Architektur provoziert, die in ihren Ausmaßen nicht abschätzbar und in ihrer Anordnung verwirrend ist. Jameson folgert aus seinen Beschreibungen, dass damit im postmodernen Raum auch die Möglichkeiten der Kritik abgeschafft worden sei: »Wir sind ab sofort in diese aufgefüllten, diffusen Räumlichkeiten so weit eingetaucht, dass unsere nunmehr postmodernen Körper der räumlichen Koordination beraubt sind: praktisch und auch theoretisch unfähig, Distanz herzustellen. $\aleph^{38}$ Diese Raumerfahrung, deren Geschichtlichkeit Jameson unterstreicht, könne in seiner bedrückenden, umfassenden Zudringlichkeit als das Modell aller postmodernen Erscheinungen figurieren.

So treffend seine Beschreibung immersiver Raumerfahrung ist, so konventionell verbleibt seine Vorstellung von Kritik. Wie bei Jameson ist auch die derzeitige Diskussion um Immersion und Atmosphärik in

36 Fredric Jameson: »Postmoderne - zur Logik der Kultur im Spätkapitalismus«, in: Andreas Huyssen/Klaus R. Scherpe (Hg.): Postmoderne. Zeichen eines kulturellen Wandels, Reinbek: Rowohlt 1986, S. 45-102, hier S. 89.

37 Ebd., S. 88.

38 Ebd., S. 94 (Hervorh. i. Orig.) 
eine falsche, simplifizierende Gegenüberstellung zur Kritik eingespannt worden. Die Aufhebung von Distanz durch das Eintauchen in den Raum lasse den notwendigen Abstand einschrumpfen, der für eine kritische Reflexion vonnöten ist. Aber weder Immersion noch räumliche Ansteckung muss Widerständigkeit und Kritik ausschließen. ${ }^{39}$ Peter Sloterdijk, der Architektur als elementare Immersionstechnik versteht, hat die Affektion durch Gebäude in einer zugespitzten Formulierung als eine Art selbst gewählter Knechtschaft oder >Wahlbesessenheit ২ bezeichnet, ohne jedoch deshalb die Möglichkeit der Kritik auszuschließen - man erlerne diese schlicht durch »Immersionswechsel «. ${ }^{40}$ Da das Bewohnen von Räumen bedeutet, ihren Atmosphären ausgeliefert zu sein, entgeht man ihnen nur, indem man Gegenräume wählt.

In jedem Fall bieten Begriffe wie Immersion und Atmosphäre Ansatzpunkte, um die von Latour anvisierte Interaktion von menschlichen und nicht menschlichen Entitäten um das Moment der Affektion zu erweitern. Als Zwischenbereich, der weder dem empfindenden Subjekt noch der Umgebung einseitig zuzuschreiben ist, bilden Atmosphären eine Sphäre der Interpassion von Mensch und Raum aus. Deren Berücksichtigung kann zum einen der Ergänzung von Latours Theorie der Hybridisierung dienen, zum anderen kann sie weitergehend dazu beitragen, das vorherrschende Handlungsparadigma, das sich auch in den heutigen Kulturwissenschaften aufweisen lässt, einer Revision zu unterziehen. Die mit dem Begriff der Interpassion angedeutete Theorie des Pathischen hätte demjenigen ihre Aufmerksamkeit zu widmen, was das Subjekt affiziert, und sie hätte dabei - der ursprünglichen zweifachen Wortbedeutung von >pathos « im Sinne von Erleiden und Empfinden folgend - sowohl für eine verstärkte Berücksichtigung von Passivität zu argumentieren als auch zu einer Neubestimmung des Gefühls beizutragen.

$39 \mathrm{Zu}$ dieser Schlussfolgerung kommt auch Ole W. Fischer, wie er anhand konkreter Beispiele der Architektur und Raumkunst u.a. von Phillippe Rahm und Diller \& Scodidio belegt: »Die atmosphärisch-immersiven Räume entziehen sich einer einfachen Zuweisung zur >kritischen` oder >post-kritischen< Theorie, indem sie Performance mit Reflexion und Engagement mit Widerstand verbinden. « Ole W. Fischer: »)Alle reden vom Wetter ...< Atmosphärische Räume zwischen kritischer Lektüre und projektiver Praxis«, in: archplus 178 (2006), S. 76-81, hier: S. 81.

40 P. Sloterdijk: Architektur als Immersionskunst, S. 58. 


\section{Literatur}

Binswanger, Ludwig: »Das Raumproblem in der Psychopathologie «, in:

Zeitschrift für Neurologie 145 (1933), S. 598-647.

Böhme, Gernot: Atmosphäre. Essays zur neuen Ästhetik, Frankfurt a.M.:

Suhrkamp 1995.

Bourdieu, Pierre: »Physischer, sozialer und angeeigneter Raum«, in:

Martin Wentz (Hg.): Stadt-Räume, Frankfurt a.M.: Campus Verlag 1991, S. 25-34.

Casey, Edward S.: »Vom Raum zum Ort in kürzester Zeit. Phänomenologische Prolegomena«, in: Phänomenologische Forschungen (2003),

S. 55-95.

Caillois, Roger: »Mimetismus et psychasthénie légendaire«, in: ders.: Le Mythe et l'homme, Paris: Edition Gallimard 1938.

De Certeau, Michel: Kunst des Handelns, Berlin: Merve 1988.

Degele, Nina/Simms, Timothy: »Bruno Latour (*1947). Post-Konstruktivismus pur«, in: Martin L. Hofmann/Tobias F. Korta/Sibylle Niekisch (Hg.): Culture Club. Klassiker der Kulturtheorie, Frankfurt a.M.: Suhrkamp 2004, S. 259-275.

Fischer, Ole W.: »Critical, Post-Critical, Projective«, in: archplus 174 (2005), S. 92-97.

Fischer, Ole W.: ») Alle reden vom Wetter ...< Atmosphärische Räume zwischen kritischer Lektüre und projektiver Praxis«, in: archplus 178 (2006), S. 76-81.

Foucault, Michel: Überwachen und Strafen. Die Geburt des Gefängnisses, Frankfurt a.M.: Suhrkamp 1989.

Foucault, Michel: »Andere Räume«, in: Karlheinz Barck u.a. (Hg.): Aisthesis. Wahrnehmung heute oder Perspektiven einer anderen Ästhetik, Leipzig: Reclam 1990, S. 34-46.

Foucault, Michel: »Das Auge der Macht«, in: ders.: Schriften, Dritter Band, Frankfurt a.M.: Suhrkamp 2003, S. 250-271.

Haraway, Donna: »Ein Manifest für Cyborgs. Feminismus im Streit mit den Technowissenschaften«, in: dies.: Die Neuerfindung der Natur. Primaten, Cyborgs und Frauen, Frankfurt a.M./New York: Campus 1995, S. 33-72.

Heidegger, Martin: Sein und Zeit, Tübingen: Niemeyer 1986.

Jameson, Fredric: »Postmoderne - zur Logik der Kultur im Spätkapitalismus«, in: Andreas Huyssen/Klaus R. Scherpe (Hg.): Postmoderne. Zeichen eines kulturellen Wandels, Reinbek: Rowohlt 1986, S. 45102.

Knorr Cetina, Karin: »Sozialität mit Objekten. Soziale Beziehungen in post-traditionalen Wissensgesellschaften«, in: Werner Rammert 
(Hg.): Technik und Sozialtheorie, Frankfurt a.M./New York: Campus 1998, S. 83-120.

Kuhnert, Nikolaus/Ngo, Anh-Linh: »Die Produktion von Präsenz. Potentiale des Atmosphärischen«, in: archplus 178 (2006), S. 22-25.

Lacan, Jacques: Das Seminar. Buch XI (1964), Berlin/Weinheim: Quadriga 1987.

Latour, Bruno: »On actor-network theory. A few clarifications «, in: Soziale Welt 47 (1996), S. 369-381.

Latour, Bruno: Wir sind nie modern gewesen. Versuch einer symmetrischen Anthropologie, Frankfurt a.M.: Fischer 1998.

Latour, Bruno: Die Hoffnung der Pandora. Untersuchungen zur Wirklichkeit der Wissenschaft, Frankfurt a.M.: Suhrkamp 2000.

Latour, Bruno: Das Parlament der Dinge. Für eine politische Ökologie, Frankfurt a.M.: Suhrkamp 2001.

Latour, Bruno: Von der Realpolitik zur Dingpolitik oder Wie man Dinge öffentlich macht, Berlin: Merve 2005.

Lefèbvre, Henri: Production de l'espace, Paris: Anthropos 1974.

Löw, Martina: Raumsoziologie, Frankfurt a.M.: Suhrkamp 2001.

Merleau-Ponty, Maurice: Phänomenologie der Wahrnehmung, Berlin: de Gruyter 1966.

Merleau-Ponty, Maurice: »Das Auge und der Geist«, in: ders.: Das Auge und der Geist. Philosophische Essays, Hamburg: Meiner 1984, S. 1343.

Minkowski, Eugène: »Ansätze zu einer Psychopathologie des Raumes«, in: ders.: Die gelebte Zeit, Bd. 2, Salzburg: Otto Müller Verlag 1972, S. $232-267$

Möntmann, Nina: Kunst als sozialer Raum, Köln: Verlag der Buchhandlung Walther König 2002.

Roselt, Jens: »Wo die Gefühle wohnen. Zur Performativität von Räumen«, in: Hajo Kurzenberger/Annemarie Matzke (Hg.): Theorie, Theater, Praxis, Berlin: Theater der Zeit 2004, S. 66-76.

Schwartz, Ullrich: »Space Body Affect«, in: Tom Fecht/Dietmar Kamper (Hg.): Umzug ins Offene. Vier Versuche über den Raum, Wien/New York: Springer 2000, S. 82-85.

Simmel, Georg: »Der Raum und die räumlichen Ordnungen der Gesellschaft«, in: ders.: Soziologie. Gesamtausgabe Bd. 11, Frankfurt a.M.: Suhrkamp 1992, S. 687-790.

Sloterdijk, Peter: »Architektur als Immersionskunst«, in: archplus 178 (2006), S. 58-61.

Straus, Erwin: »Die Formen des Räumlichen«, in: ders.: Psychologie der menschlichen Welt, Berlin u.a.: Springer Verlag 1960, S. 141-178. 
